\section{Pentraxin-3 polymorphisms and invasive mold infections in acute leukemia patients receiving intensive chemotherapy}

Invasive mold infections are a major concern in oncohematologic patients, with incidence and mortality rates ranging between $15-30 \% .{ }^{1}$ While mold-active prophylaxis is recommended during induction chemotherapy for acute leukemia, its universal use has been challenged based on the large number of patients needed to treat, ${ }^{2-4}$ associated costs, toxicities, and the emergence of azoleresistance. ${ }^{5}$ Pre-emptive strategies combining serum galactomannan monitoring and computed tomography represent an alternative approach. However, there is an urgent need for reliable markers of susceptibility to invasive mold infections allowing for risk-stratified prophy-

Table 1. Patients' characteristics.

\begin{tabular}{|c|c|c|c|c|}
\hline Characteristics (proportion)a & $\begin{array}{c}\text { All } \\
\text { Episodes } \\
\mathrm{N}=212\end{array}$ & $\begin{array}{l}\text { No Invasive } \\
\text { Mold Infection } \\
\text { N=186 }\end{array}$ & $\begin{array}{c}\text { Invasive } \\
\text { Mold Infection } \\
\text { N=26 }\end{array}$ & $\begin{array}{c}P \\
(\log \text { rank) }\end{array}$ \\
\hline Median age, years [IQR $]$ & $54[21]$ & $55[20]$ & $51[28]$ & 0.6 \\
\hline Median weight, $\mathrm{kg}\left[\mathrm{IQR}^{\mathrm{b}}\right]$ & $73[19]$ & $73[19]$ & $75[23]$ & 0.5 \\
\hline Sex, male & $130(0.61)$ & $116(0.62)$ & $14(0.54)$ & 0.4 \\
\hline Ethnicity, other than Caucasian ${ }^{c}$ & $9(0.04)$ & $8(0.04)$ & $1(0.04)$ & 0.5 \\
\hline \multicolumn{5}{|l|}{ Hematologic malignancy } \\
\hline Acute myeloid leukemia & $150(0.71)$ & $134(0.72)$ & $16(0.62)$ & Ref. \\
\hline Acute lymphoblastic leukemia & $45(0.21)$ & $40(0.22)$ & $5(0.19)$ & 0.9 \\
\hline Refractory anemia with excess blast type 2 & $17(0.08)$ & $12(0.06)$ & $5(0.19)$ & 0.02 \\
\hline Preexisting neutropenia ${ }^{\mathrm{d}}$ at leukemia diagnosis & $79(0.37)$ & $68(0.37)$ & $11(0.44)$ & 0.6 \\
\hline \multicolumn{5}{|l|}{ Previous HSCT } \\
\hline Allogeneic ${ }^{\mathrm{e}}$ & $9(0.04)$ & $7(0.04)$ & $2(0.08)$ & 0.3 \\
\hline Autologous & $4(0.02)$ & $3(0.02)$ & $1(0.04)$ & 0.3 \\
\hline \multicolumn{5}{|l|}{ Relapsed disease } \\
\hline As reason for admission ${ }^{f}$ & $25(0.12)$ & $22(0.12)$ & $3(0.12)$ & 1.0 \\
\hline During follow-up & $15(0.07)$ & $13(0.07)$ & $2(0.08)$ & 1.0 \\
\hline Reinduction chemotherapy & $20(0.09)$ & $17(0.09)$ & $3(0.12)$ & 0.7 \\
\hline \multicolumn{5}{|l|}{ Comorbidities } \\
\hline Cardiac insufficiency & $29(0.14)$ & $26(0.14)$ & $3(0.12)$ & 0.7 \\
\hline Chronic obstructive pulmonary disease & $26(0.12)$ & $21(0.11)$ & $5(0.19)$ & 0.3 \\
\hline Diabetes mellitus & $20(0.09)$ & $17(0.09)$ & $3(0.12)$ & 0.6 \\
\hline Neurologic disease & $12(0.06)$ & $12(0.06)$ & & \\
\hline Chronic renal insufficiency & $5(0.02)$ & $3(0.02)$ & $2(0.08)$ & 0.07 \\
\hline Positive CMV serostatus & $138(0.65)$ & $123(0.66)$ & $15(0.58)$ & 0.4 \\
\hline \multicolumn{5}{|l|}{ Anti-mold prophylaxis } \\
\hline Initial hospital stay (e.g. induction) & $20(0.09)$ & $19(0.10)$ & $1(0.04)$ & 0.3 \\
\hline $\begin{array}{l}\text { Throughout chemotherapy course } \\
\text { (>80\% neutropenic days) }\end{array}$ & $12(0.06)$ & $11(0.06)$ & $1(0.04)$ & 0.7 \\
\hline Invasive mold infections & $26(0.12)$ & & $26(1.00)$ & \\
\hline Probable & $16(0.08)$ & & $16(0.62)$ & \\
\hline Proven & $10(0.05)$ & & $10(0.38)$ & \\
\hline \multicolumn{5}{|l|}{ Organism } \\
\hline Aspergillus spp. & $20(0.09)$ & & $20(0.77)$ & \\
\hline Mucorales & $4(0.02)$ & & $4(0.15)$ & \\
\hline Other/mixed ${ }^{\mathrm{h}}$ & $2(0.01)$ & & $2(0.08)$ & \\
\hline \multicolumn{5}{|l|}{ Localization } \\
\hline Respiratory tract ${ }^{i}$ & $24(0.11)$ & & $24(0.92)$ & \\
\hline Other & $2(0.01)$ & & $2(0.08)$ & \\
\hline
\end{tabular}

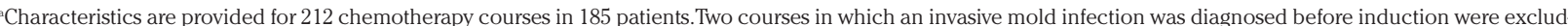
ed. ${ }^{\circ} P$ value was obtained by using stcrreg (continuous variables). ${ }^{\circ}$ Ethnicities other than Caucasian included South American ( $\mathrm{n}=5$ ), Asian ( $\mathrm{n}=3$ ) and African ( $\mathrm{n}=2$ ).

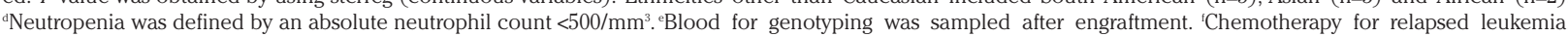
${ }^{8}$ Chemotherapy cycles included induction, consolidation and potential reinduction during a period of 6 months ${ }^{\mathrm{h}}$ One infection was due to Mucorales with Aspergillus spp., and one to Hormographiella aspergillata. Twenty-two had a pulmonary infection and two a sinusal infection. 'One patient with a cerebral abscess and one with a colic infection. IQR, interquartile range; HSCT: hematopoietic stem cell transplant; CMV: cytomegalovirus. 
lactic strategies that combine optimal prevention with limited exposure to drugs. ${ }^{6,7}$

Pentraxin-3 (PTX3) is a pattern recognition receptor that is important for the innate immune detection of several pathogens; by binding to Aspergillus conidia, it facilitates opsonization as well as complement and phagocyte activation. ${ }^{8}$ Single nucleotide polymorphisms leading to PTX3 deficiency have been identified as strong predictors of invasive aspergillosis in recipients of allogeneic hematopoietic stem cell transplants ${ }^{9,10}$ and solid organ transplants. ${ }^{11,12}$ In the present study, we investigated the role of two PTX3 single nucleotide polymorphisms for susceptibility to invasive mold infections in patients with acute leukemia undergoing intensive chemotherapy.

All adult patients hospitalized in the Isolation Unit of the Lausanne University Hospital for acute myeloid leukemia (AML), acute lymphoblastic leukemia (ALL), or refractory anemia with excess blasts-2 (RAEB-2) between 2007 and 2017 who signed informed consent to genetic biobanking were included. The study protocol was approved by the local Ethics Committee (Swissethics 2017-01975). Clinical data were prospectively collected during chemotherapy courses. Patients with AML or RAEB-2 received a first cycle of cytarabine $\left(200 \mathrm{mg} / \mathrm{m}^{2}\right.$ over 7 days) with idarubicin $\left(12 \mathrm{mg} / \mathrm{m}^{2}\right)$ or daunorubicin $\left(60 \mathrm{mg} / \mathrm{m}^{2}\right)$ over 3 days ${ }^{13}$ and a second cycle with highdose cytarabine, according to ongoing HOVON/SAKK protocols. Patients with ALL were treated according to the GRAALL 2005 protocol. ${ }^{14}$ Neutropenia was defined by an absolute neutrophil count $<500 \mathrm{cells} / \mathrm{mm}^{3}$. Proven or probable invasive mold infections were diagnosed according to standard definitions. ${ }^{2}$ Routine anti-mold prophylaxis is not administered in leukemia patients treated with chemotherapy at our center, but a minority of patients may receive such therapy at the discretion of the treating physician (e.g. those with a previous invasive mold infection, high-risk environmental exposure, prolonged high-dose corticosteroid therapy and prolonged neutropenia before induction, or immediate reinduction for early relapse without hematopoietic recovery). Patients with relapsing leukemia after allogeneic hematopoietic stem cell transplant were considered only if samples for genotyping were obtained after engraftment. Two single nucleotide polymorphisms in PTX3 (rs2305619 and rs3816527) were genotyped using a competitive allele-specific polymerase chain reaction assay (KASPTM, LGC Genomics, UK).

The statistical analysis was performed using STATA version 14.2 software (StataCorp LP, College Station, TX, USA). The association between invasive mold infections and PTX3 single nucleotide polymorphisms was assessed by 6 -month cumulative incidence curves and uni- and multivariable regression models using the stcrreg program implemented in Stata, starting from the first day of induction therapy, with censoring on the last hospitalization date, considering death or hematopoietic stem cell transplant as a competing risk. Linkage disequilibrium and Hardy-Weinberg equilibrium were assessed using the pwld and hwe programs implemented in Stata. PTX3 haplotypes were generated using PHASE version 2.1 (University of Washington, Seattle, WA, USA). The statistical power was calculated using the powerSurvEpi package 0.0.9 in R ( $\mathrm{R}$ Core Team, Vienna, Austria).

Among 185 patients included, 159 patients (126 with AML/RAEB-2 and 33 with ALL) received one chemotherapy regimen according to AML and ALL protocols, and 26 patients (20 with AML/RAEB-2 and 6 with ALL) received more than one regimen (associating the initial regimen and salvage therapy for a relapse at least 6 months after first complete remission), for a total of 212 chemotherapy regimens (150 in AML, 45 in ALL and 17 in RAEB-2 patients). A minority ( $9 \%$ ) had mold-active prophylaxis at induction. The median age of the patients was 55 years and $61 \%$ were male (Table 1 ). A total of 26 invasive mold infections (6-month cumulative incidence $=14 \%$ ) occurred, including 16 probable and 10 proven infections. Twenty were due to Aspergillus species $(77 \%)$, four to Mucorales (15\%) and two to other/mixed pathogens $(8 \%)$. Most of the invasive mold infections were located within the respiratory tract $(n=24,92 \%)$. In the univariate analysis, the single clinical factor associat-
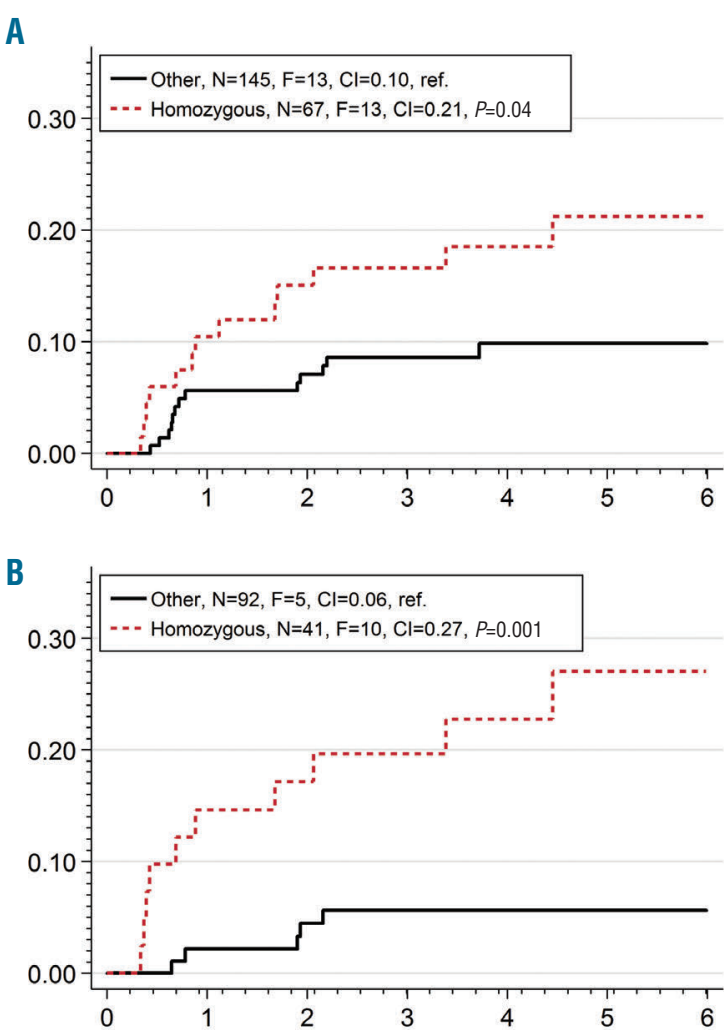

C

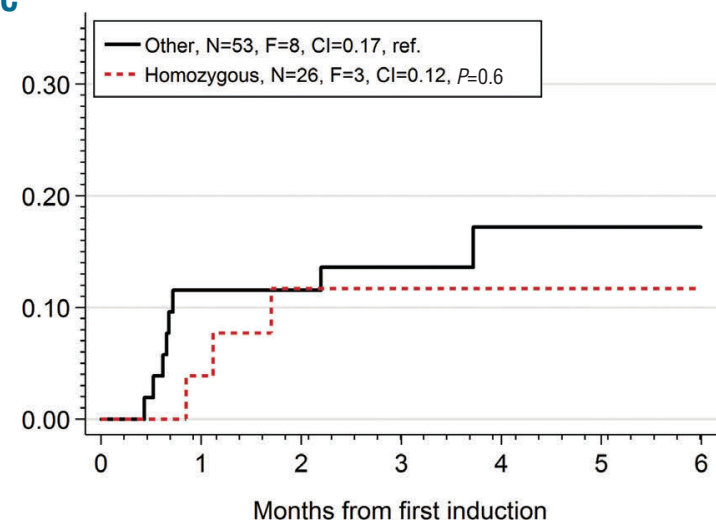

Figure 1. Cumulative incidence of probable/proven invasive mold infections according to homozygosity for rs2305619 and/or rs3816527 PTX3 single nucleotide polymorphisms. (A) All chemotherapy courses $(n=212)$. (B) Chemotherapy courses starting in patients without pre-existing neutropenia $(n=133)$. (C) Chemotherapy courses starting in patients with pre-existing neutropenia $(n=79)$. The $P$ value stands for the log-rank test. N: number of chemotherapy cycles at risk; F: number of failures (i.e. invasive mold infections); $\mathrm{Cl}$ : 6-month cumulative incidence of invasive mold infections. 
ed with the risk of invasive mold infection was having RAEB-2 rather than AML $(P=0.02)$.

The PTX3 rs3816527 and rs2305619 single nucleotide polymorphisms had minor allele frequencies of 0.44 and 0.48 , respectively, with a pairwise linkage disequilibrium $\left(R^{2}\right)$ value of 0.78 , and both were in Hardy-Weinberg equilibrium. The 6-month cumulative incidence of invasive mold infections was $21 \%$ for patients carrying two copies of the minor allele of PTX3 single nucleotide polymorphisms rs2305619 and/or rs3816527 compared to $10 \%$ for patients without these single nucleotide polymorphisms (log-rank test $P=0.04$ ) (Figure 1A). When patients were stratified based on their absolute neutrophil count at the time of leukemia diagnosis, the association was significant among patients without pre-existing neutropenia (absolute neutrophil count $\geq 500 / \mathrm{mm}^{3}$, cumulative incidence $27 \%$ versus $6 \% ; P=0.001$ ) (Figure 1B), but not among those admitted with neutropenia (absolute neutrophil count $<500 / \mathrm{mm}^{3}$, cumulative incidence $12 \%$ versus $17 \% ; P=0.6$ ) (Figure $1 \mathrm{C}$ ). Multivariable analyses confirmed a significant interaction between PTX3 single nucleotide polymorphisms and pre-existing neutropenia $(P=0.04)$ (Online Supplementary Table S1). Among patients without pre-existing neutropenia, homozygosity for the minor allele of PTX3 rs2305619 and/or $r s 3816527$ was an independent predictor of invasive mold infections (hazard ratio $=5.06,95 \%$ confidence interval 1.68-15.2; $P=0.004$ ), after adjustment for the type of underlying disease.

Similar trends were observed when patients were stratified according to type of underlying hematologic malignancy (AML/RAEB-2 versus ALL) and previous receipt of an allogeneic hematopoietic stem cell transplant (Online Supplementary Figure S1). Moreover, associations were similar, though slightly less significant, when analyses were performed for the carriage of two copies of the minor alleles of both rs2305619 and rs3816527 (instead of any) or when each single nucleotide polymorphism was considered separately (Online Supplementary Figure S2). The associations remained or tended to remain significant after exclusion of courses with anti-mold prophylaxis, after excluding patients with a possible invasive mold infection (rather than considering them as controls), and after excluding patients with invasive mold infections due to non-Aspergillus molds (Online Supplementary Figure S3).

To our knowledge, this is the first study to demonstrate an association between PTX3 single nucleotide polymorphisms and invasive mold infections in patients with acute leukemia. Previous studies showed robust associations between these polymorphisms and invasive mold infections among both hematopoietic stem cell transplant recipients ${ }^{9,10}$ and solid organ transplant recipients. ${ }^{11,12}$ The strength of these associations, together with the relative high allele frequency of the single nucleotide polymorphisms and replication among different at-risk population have made PTX3 single nucleotide polymorphisms the most reliable marker of invasive mold infections in these populations.

Previous investigators reported no association between PTX3 single nucleotide polymorphisms and invasive aspergillosis in a cohort of 153 non-transplanted hematology patients with prolonged neutropenia. ${ }^{9}$ The apparent discrepancy with our observation may stem from the fact that patients in the previous study were not stratified according to their initial absolute neutrophil count. We found a significant interaction between PTX3 single nucleotide polymorphisms and pre-existing neutropenia, suggesting that such stratification is a key factor to be accounted for. Nevertheless, the reason why PTX3 single nucleotide polymorphisms do not seem to influence susceptibility to invasive mold infections in patients with acute leukemia and pre-existing neutropenia, in contrast to those without pre-existing neutropenia, as well as those who have undergone hematopoietic stem cell or solid organ transplantation, which usually do not initially present with neutropenia, are unknown. Considering the pivotal role of neutrophils in the production and storage of PTX $3,{ }^{15}$ patients without neutropenia upon presentation may retain a sufficient level of the PTX3 protein at the time of induction, thereby maintaining sufficient protection during the neutropenic phase and until neutrophil recovery. In contrast, patients with pre-existing neutropenia may progressively develop alternative immune mechanisms, making them susceptible to invasive mold infections independently of PTX3 polymorphisms.

Broad-spectrum azoles active against filamentous molds were endorsed by international guidelines as the standard of care for antifungal prophylaxis in patients at high risk of invasive mold infections, ${ }^{2}$ but universal prophylaxis is not routinely administered in some centers with a relatively low incidence of invasive mold infections, ${ }^{3}$ except for hematopoietic stem cell transplant recipients. In the light of our results on PTX3, genetically-targeted antifungal prophylaxis would be particularly useful in patients undergoing intensive chemotherapy for acute leukemia, to whom universal prophylaxis is not always given, even though invasive aspergillosis remains more frequent in such patients than in solid organ transplant recipients. ${ }^{11,12}$

Our study is limited by its sample size, which prevented testing of a large number of polymorphisms. Nevertheless, given the high minor allele frequencies of PTX3 single nucleotide polymorphisms, the study had $80 \%$ power to detect an effect size of 2.0 or more. In our study, the associations were more significant when homozygosity for either of the single nucleotide polymorphisms rs2305619 or rs3816527, was present. Cunha's study among hematopoietic stem cell transplant recipients showed a more significant association when both single nucleotide polymorphisms were present together (h2/h2 diplotypes), ${ }^{6}$ but subsequent studies found a more significant association for $r s 2305619^{10}$ and rs3816527 $7^{11}$ taken separately. Given the high level of linkage disequilibrium between the two single nucleotide polymorphisms, it is difficult to determine whether homozygosity for either of them, or both, is clinically relevant.

In conclusion, the new evidence for a robust association between PTX3 single nucleotide polymorphisms and invasive mold infections among patients with acute leukemia receiving intensive chemotherapy makes PTX3 one of the most promising markers for novel management strategies, especially in this population which may be particularly well suited for genetically-targeted antifungal prophylaxis. While the association between PTX3 single nucleotide polymorphisms among patients with acute leukemia is fully consistent with previous observations among other at-risk populations, additional studies are necessary to elucidate the role of neutropenia at presentation and its interaction with the PTX3 single nucleotide polymorphisms.

Anne-Sophie Brunel, 1" Agnieszka Wójtowicz, ${ }^{*}$ Frédéric Lamoth, ${ }^{1,2}$ Olivier Spertini, ${ }^{3}$ Dionysios Neofytos, ${ }^{4}$ Thierry Calandra, ${ }^{1}$ Oscar Marchetti ${ }^{1,5}$ and Pierre-Yves Bochud

'Infectious Diseases Service, Department of Medicine, Lausanne University Hospital (CHUV); ${ }^{2}$ Microbiology Institute, Lausanne 
University Hospital (CHUV; ${ }^{3}$ Hematology Service, Department of Oncology, Lausanne University Hospital (CHUV); Infectious

Diseases Service, University Hospital Geneva (HUG) and

'Department of Medicine, Ensemble Hospitalier de la Côte, Morges, Switzerland.

*ASB and $A W$ contributed equally to this work.

Correspondence:Pierre-Yves.Bochud@chuv.ch doi:10.3324/haematol.2018.195453

Information on authorship, contributions, and financial \& other disclosures was provided by the authors and is available with the online version of this article at www. haematologica.org.

\section{References}

1. Kontoyiannis DP, Marr KA, Park BJ, et al. Prospective surveillance for invasive fungal infections in hematopoietic stem cell transplant recipients, 2001-2006: overview of the Transplant-Associated Infection Surveillance Network (TRANSNET) database. Clin Infect Dis. 2010; 50(8):1091-1100.

2. De Pauw B, Walsh TJ, Donnelly JP, et al. Revised definitions of invasive fungal disease from the European Organization for Research and Treatment of Cancer/Invasive Fungal Infections Cooperative Group and the National Institute of Allergy and Infectious Diseases Mycoses Study Group (EORTC/MSG) Consensus Group. Clin Infect Dis. 2008;46(12):1813-1821.

3. De Pauw BE, Donnelly JP. Prophylaxis and aspergillosis--has the principle been proven? N Engl J Med. 2007;356(4):409-411.

4. Cornely OA, Ullmann AJ. Numbers needed to treat with posaconazole prophylaxis to prevent invasive fungal infection and death. Clin Infect Dis. 2008;46(10):1626-1627.
5. Chowdhary A, Sharma C, Meis JF. Azole-resistant aspergillosis: epidemiology, molecular mechanisms, and treatment. J Infect Dis. 2017;216(suppl_3):S436-S444.

6. Cunha C, Aversa F, Romani L, et al. Human genetic susceptibility to invasive aspergillosis. PLoS Pathog. 2013;9(8):e1003434.

7. Wójtowicz A, Bochud PY. Host genetics of invasive Aspergillus and Candida infections. Semin Immunopathol. 2015;37(2):173-186.

8. Garlanda C, Hirsch E, Bozza S, et al. Non-redundant role of the long pentraxin PTX3 in anti-fungal innate immune response. Nature. 2002;420(6912):182-186.

9. Cunha C, Aversa F, Lacerda JF, et al. Genetic PTX3 deficiency and aspergillosis in stem-cell transplantation. $\mathrm{N}$ Engl $\mathrm{J}$ Med. 2014;370(5):421-432.

10. Fisher CE, Hohl TM, Fan W, et al. Validation of single nucleotide polymorphisms in invasive aspergillosis following hematopoietic cell transplantation. Blood. 2017;129(19):2693-2701.

11. Wójtowicz A, Lecompte TD, Bibert S, et al. PTX3 polymorphisms and invasive mold infections after solid organ transplant. Clin Infect Dis. 2015;61(4):619-622.

12. Cunha C, Monteiro AA, Oliveira-Coelho A, et al. PTX3-based genetic testing for risk of aspergillosis after lung transplant. Clin Infect Dis. 2015;61(12):1893-1894.

13. Lowenberg B, Pabst T, Maertens J, et al. Therapeutic value of clofarabine in younger and middle-aged (18-65 years) adults with newly diagnosed AML. Blood. 2017;129(12):1636-1645.

14. Maury S, Chevret S, Thomas X, et al. Rituximab in B-lineage adult acute lymphoblastic leukemia. N Engl J Med. 2016;375(11):10441053.

15. Jaillon S, Peri G, Delneste Y, et al. The humoral pattern recognition receptor PTX3 is stored in neutrophil granules and localizes in extracellular traps. J Exp Med. 2007;204(4):793-804. 